\title{
Rotura esplénica atraumática secundaria a perforación apendicular por vólvulo de ciego
}

\author{
Atraumatic splenic rupture secondary to appendicular perforation by cecal volvulus
}

Alejandro Menéndez-Moreno*, Mauricio Burneo-Esteves, Rafael Orti-Rodríguez, Ana Feria-González, Antonio Pérez-Álvarez y Manuel Barrera-Gómez

Servicio de Cirugía General y del Aparato Digestivo, Hospital Universitario Nuestra Señora de Candelaria, Santa Cruz de Tenerife, Tenerife, España

\begin{abstract}
Resumen
La rotura esplénica atraumática, una emergencia quirúrgica infrecuente, constituye una situación de riesgo vital. La falta de antecedente traumático dificulta su sospecha. Presentamos el caso de una mujer de 45 años, con antecedente de cirugía bariátrica, remitida a nuestro servicio de urgencias por dolor abdominal y lumbar de 3 semanas, en shock. Con el diagnóstico imagenológico de rotura esplénica, se realizó cirugía emergente, con hallazgos atípicos, y la paciente tuvo una buena evolución. La revisión bibliográfica, los antecedentes y los hallazgos quirúrgicos permitieron postular la causalidad en esta inusual presentación. Pese a su poca frecuencia, esta patología comporta una mortalidad importante y debe tenerse presente ante un paciente en shock en la sala de urgencias.
\end{abstract}

PALABRAS CLAVE: Abdomen. Bazo. Rotura espontánea. Vólvulo intestinal. Apendicitis.

\begin{abstract}
Atraumatic splenic rupture, an infrequent surgical emergency, constitutes a life-threatening situation. The lack of a traumatic background makes it difficult to suspect. We present a case of a 45-year-old woman, with history of bariatric surgery, referred to our emergency due to 3 weeks long abdominal and lumbar pain, in shock. Imaging diagnosis of splenic rupture, emergent surgery was performed with atypical findings and good evolution. The bibliographic review, background and surgical findings allowed to postulate causality in this unusual presentation. Despite its low frequency, this pathology involves significant mortality and must be present in the patient in shock in the emergency room.
\end{abstract}

KEY WORDS: Abdomen. Spleen. Spontaneous rupture. Intestinal volvulus. Appendicitis.

\section{Introducción}

La rotura esplénica atraumática es una emergencia quirúrgica poco común que implica dificultades diagnósticas considerables y que representa un riesgo vital para el paciente en la sala de urgencias. La diversidad de causas posibles hace más difícil dar con el diagnóstico, por lo que un alto grado de sospecha y un manejo clínico adecuado son cruciales para su adecuada resolución. Se presenta un caso de rotura esplénica atraumática desencadenada por una perforación apendicular debida a un vólvulo de ciego, con su manejo y desenlace, por ser infrecuente.

\author{
Correspondencia: \\ *Alejandro Menéndez-Moreno \\ Mencey Zebenzuy, 10, puerta 2i \\ C.P. 38108 , San Cristóbal de La Laguna, \\ Tenerife, España \\ E-mail: amenendez88@gmail.com
}

Fecha de recepción: 18-04-2018

Fecha de aceptación: 05-06-2018

DOI: $10.24875 / C I R U .18000373$
Cir Cir. 2019;87:211-214

Contents available at PubMed www.cirugiaycirujanos.com 


\section{Presentación del caso}

Mujer de 45 años con antecedentes quirúrgicos de histerectomía, bypass gastroyeyunal laparoscópico 4 años antes con pérdida de $57 \mathrm{~kg}$ y colecistectomía laparoscópica posterior que acude a un centro externo por dolor lumbar y abdominal tipo cólico de 3 semanas de evolución. Niega concomitantes y antecedente traumático alguno. Se realiza tomografía computarizada que reporta rotura esplénica, vólvulo de ciego, perforación apendicular y líquido libre intraabdominal (Figs. 1 a 3), y es remitida al servicio de urgencias de nuestra institución. A su llegada se encuentra hemodinámicamente inestable (presión arterial $86 / 39 \mathrm{mmHg}$, frecuencia cardíaca 110 latidos por minuto) y la exploración física revela una puñopercusión izquierda positiva y abdomen agudo. En la analítica se hace patente un patrón de respuesta inflamatoria (Tabla 1) con frotis de sangre periférica patológico. Dados los hallazgos, se decide iniciar antibioticoterapia de amplio espectro y realizar cirugía urgente.

Durante la intervención, tras acceder por laparotomía media, se evidencia hemoperitoneo de 2 litros, vólvulo cecal en el hipocondrio izquierdo con desplazamiento de asas de intestino delgado al hemiabdomen derecho, rotura de la mitad superior del bazo con sangrado activo, cápsula piógena (Fig. 4) que compromete el bazo y el estómago (remanente y excluido), así como perforación de la base apendicular (Fig. 5). Las anastomosis se encuentran indemnes. Tras el aspirado del hemoperitoneo se realiza desvolvulación del ciego con salida de abundante contenido purulento, esplenectomía, resección ileocecal (Fig. 6) con anastomosis ileocólica y lavado exhaustivo de la cavidad.

Los hallazgos descritos son confirmados por el servicio de anatomía patológica. En el cultivo de las muestras operatorias se aíslan Escherichia coli y Streptococcus anginosus, ambos cubiertos por la antibioticoterapia inicial. En el posoperatorio presenta una colección en celda esplénica que se drena por vía percutánea. Por su evolución satisfactoria, es dada de alta a los 15 días de la cirugía, previa administración de profilaxis posesplenectomía y control en consultas externas, con buen estado general, y un año después se encuentra asintomática.

\section{Discusión}

La rotura esplénica es una complicación ampliamente descrita del traumatismo abdominal, siendo menos común la rotura atraumática, que en la mayoría de los

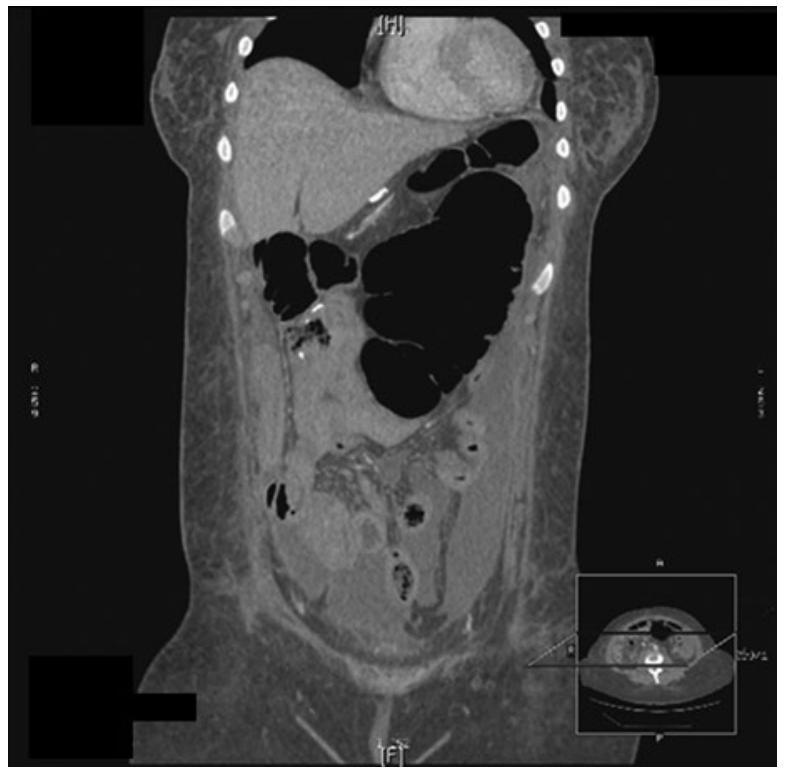

Figura 1. Tomografía computarizada (corte coronal). Se evidencia el desplazamiento del ciego al hipocondrio izquierdo.

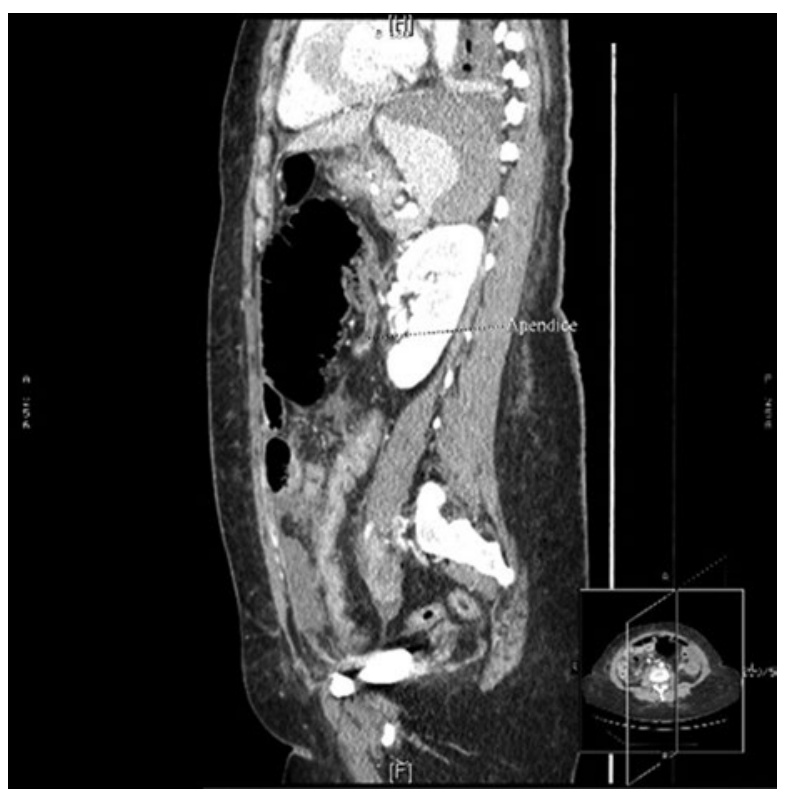

Figura 2. Tomografía computarizada (corte sagital). Se aprecian dilatación de ciego, apendicolitos y hematoma subcapsular esplénico.

casos $(90 \%)$ se desarrolla sobre un bazo afecto, ya sea por infecciones, coagulopatías o neoplasias como causas principales ${ }^{1}$. El absceso esplénico es en particular raro, con una incidencia reportada del $0.05-0.7 \%$, y es sumamente inusual que resulte en rotura del bazo ${ }^{2}$.

Por otra parte, el vólvulo de ciego es el segundo tipo más común de vólvulo colónico después del de sigma (15 y $80 \%$, respectivamente), y se relaciona con el meso redundante y el sexo femenino, factores ambos presentes en nuestra paciente. Esta torsión de un segmento colónico sobre su eje mesentérico puede 


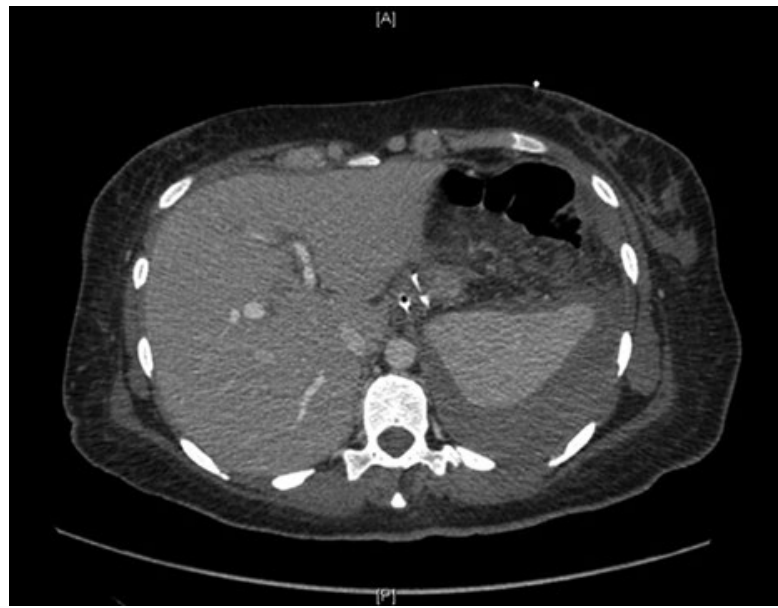

Figura 3. Tomografía computarizada (corte axial). Se observan dilatación de ciego, colección líquida y hematoma subcapsular esplénico.

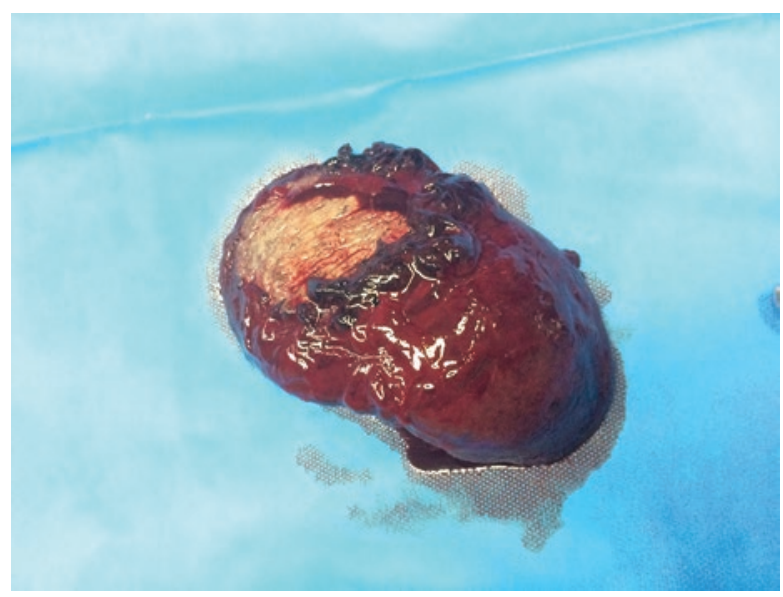

Figura 4. Pieza de esplenectomía. Se observan la cápsula del absceso y restos del hematoma coagulado.

Tabla 1. Pruebas de laboratorio al ingreso

\begin{tabular}{lc}
\hline Hemograma & \\
Hemoglobina & $12.8 \mathrm{~g} / \mathrm{dl}$ \\
Hematocrito & $38.1 \%$ \\
Leucocitos & $9000 / \mu \mathrm{l}$ \\
Neutrófilos & $86.5 \%$ \\
Plaquetas & $20,000 / \mu \mathrm{l}$ \\
Bioquímica & \\
Glucosa & $166 \mathrm{mg} / \mathrm{dl}$ \\
Creatinina & $1.05 \mathrm{mg} / \mathrm{dl}$ \\
Proteía C reactiva & $27.48 \mathrm{mg} / \mathrm{dl}$ \\
Procalcitonina & $55.71 \mathrm{ng} / \mathrm{ml}$ \\
Coagulación & \\
Tiempo de protrombina & $14.1 \mathrm{~s}$ \\
Tiempo parcial de tromboplastina activada & $70.4 \mathrm{~s}$ \\
\hline
\end{tabular}

provocar el compromiso vascular del asa intestinal, con desarrollo de gangrena y perforación de su pared $^{3}$, y se ha descrito como causa de dolor abdominal en el posoperatorio de bypass gastroyeyunal ${ }^{4}$.

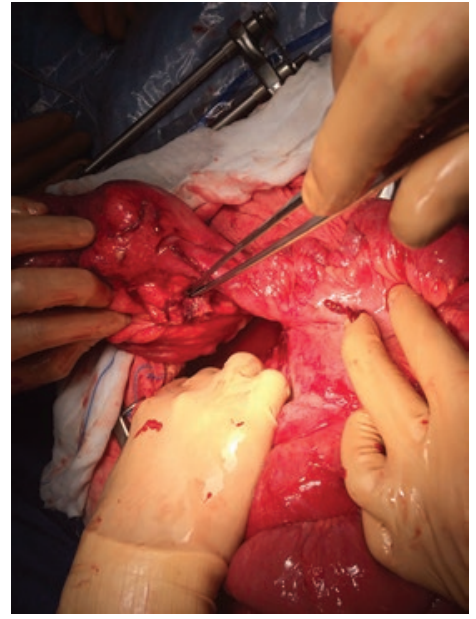

Figura 5. Vólvulo cecal in situ. Perforación en la base del apéndice cecal señalada con la pinza.

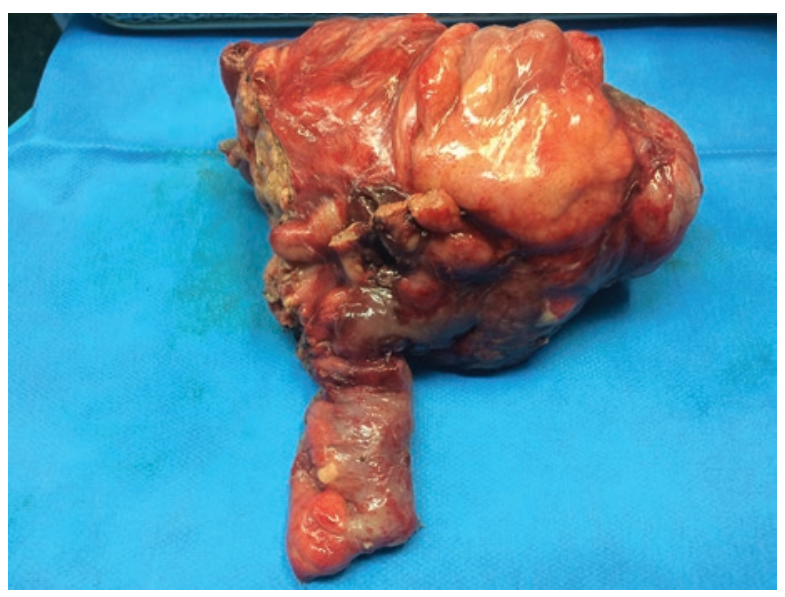

Figura 6. Pieza de resección ileocecal. Se observan la dilatación y parte de la cápsula del absceso.

Lo expuesto, en conjunto con los hallazgos por la imagen e intraoperatorios, nos hacen plantear como secuencia fisiopatológica de este atípico caso que el antecedente de cirugía bariátrica con la reducción de peso y la laxitud de los mesos intestinales resultantes permitieron una movilidad mayor del ciego, con su consecuente volvulación que pudo generar un aumento retrógrado de la presión en la luz en tubo digestivo que derivó en la rotura de la pared en el punto de menor resistencia, en este caso la base apendicular, que por su localización anómala en el hipocondrio izquierdo produjo una infección local adyacente al bazo por flora normal que afectó a dicho órgano con su consecuente rotura, dando el cuadro clínico de la paciente.

\section{Conclusiones}

A pesar de su escasa frecuencia $(0.1-0.5 \%)^{5}$, la rotura esplénica atraumática condiciona una mortalidad 
de hasta un $12.2 \%$, siendo las principales causas neoplásicas $(30.3 \%)$, infecciosas $(27.3 \%)$ y estados inflamatorios no infecciosos $(20.0 \%)^{2}$. En nuestro caso se trata de una rotura esplénica por causa infecciosa no hematógena, que presenta una peculiar sumatoria de sucesos, en una paciente cuya reducción de peso favorece la volvulación del ciego y esta a su vez una perforación apendicular, resultando en una infección localizada que involucra al bazo y finalmente condiciona su rotura. Demostramos, por medio de la historia clínica y los hallazgos físicos atípicos asociados retrospectivamente mediante una secuencia causal lógica, la necesidad de tener en cuenta como causa etiopatogénica de rotura esplénica todas aquellas infecciones intraabdominales que puedan comprometer el bazo.

\section{Financiación}

Los autores declaran no haber recibido financiación para la realización de este trabajo.

\section{Conflicto de intereses}

Los autores declaran no tener ninguna relación financiera ni personal con otras personas u organizaciones que pudieran dar lugar a conflicto de intereses.

\section{Responsabilidades éticas}

Protección de personas y animales. Los autores declaran que para esta investigación no se han realizado experimentos en seres humanos ni en animales.

Confidencialidad de los datos. Los autores declaran que han seguido los protocolos de su centro de trabajo sobre la publicación de datos de pacientes.

Derecho a la privacidad y consentimiento informado. Los autores han obtenido el consentimiento informado de los pacientes y/o sujetos referidos en el artículo. Este documento obra en poder del autor de correspondencia.

\section{Bibliografía}

1. Tonolini M, lerardi AM, Carrafiello G. Atraumatic splenic rupture, an underrated cause of acute abdomen. Insights Imaging. 2016;7:641-6.

2. Patel R, Pai A, Al-Shoek I, Evans C, Gordon A. Splenic abscess rupture post appendicectomy. BMJ Case Rep. 2012. pii: bcr0120125705.

3. Rodríguez-Hermosa JI, Martín A, Farrés R, Pont J, Codina-Cazador A, Ruiz B, et al. Obstrucción intestinal por vólvulo de ciego. Cirugía Española. 2005;78:385-7.

4. Sujka J, Teixeira AF, Jawad MA. Suspected cecal volvulus as a reason for abdominal pain after gastric bypass: a case series. Surg Obes Relat Dis. 2015;11:978-9.

5. Kocael PC, Simsek O, Bilgin IA, Tutar O, Saribeyoglu K, Pekmezci S, et al. Characteristics of patients with spontaneous splenic rupture. Int Surg. 2014;99:714-8. 